\title{
INCIDENCE OF BERTOLOTTI'S SYNDROME IN LUMBOSACRAL SURGERY PROCEDURES
}

Raafat Kamal Ragab, Mahmoud El Sayed Mohammed Nafady, Ahmed Gamal Basiony Ashour Department of Orthopaedic Surgery and Traumatology, Faculty of Medicine, Alexandria University

\section{Introduction}

Bertolotti's syndrome (BS) describes the relationship between low back pain (LBP) and lumbosacral transitional vertebra (LSTV). It's a factor that's sometimes overlooked when it comes to evaluating and treating lower back pain. The LSTV is categorised into several anatomic positions and types. Because of the different diagnostic modalities and criteria used in the research, the LSTV incidence in the general population varies greatly, and hence the link between LSTV and LBP remains contentious.

It has been suggested that the source of low back pain could be caused by disruption of biomechanics of the lowest vertebral segment of the lumbar spine produced by a unilateral aberrant articulation. Moreover, it has been proposed that biomechanical stress applied to the upper mobile vertebral segment can hasten early disc degeneration at nearby levels, resulting in disc protrusion or extrusion, which leads to back pain and sciatic pain.

\section{Aim of the work}

This work aimed to assess the incidence of Bertolotti's syndrome among laminectomy, foraminectomy, micronucleotomy and PLIF patients.

\section{Subjects and Methods}

\section{PATIENTS:}

A total number of 288 patients who undergone lumbosacral surgical procedures between January 2016 and may 2020 were considered for inclusion in this study. All cases were done by the same surgical team at El Hadra University Hospital Spine Unit.

The patients were divided into two groups. Group A consisted of patients in which LSTV was detected by radiologic findings. In contrast, Group B was formed of patients in which LSTV was not detected. 133 patients in group A and 155 in group B were analyzed.

\section{METHODS:}

Radiologic Diagnosis of LSTV

Preoperatively, all patients did plain radiographs and MRI. The presence of LSTV is best seen on Ferguson view which is a true $30^{\circ}$ angled AP radiograph of lumbosacral junction

Method of assessment of results:

The primary outcome measure was pain intensity. Patients completed the same questionnaire preoperatively and postoperatively at each follow-up visit. The questionnaire consists of a 10-point VAS for both LBP and leg pain Secondary outcome measure included the ODI. Follow-up visits occurred at 3 , 6 , and 12 months postoperatively

\section{Results}

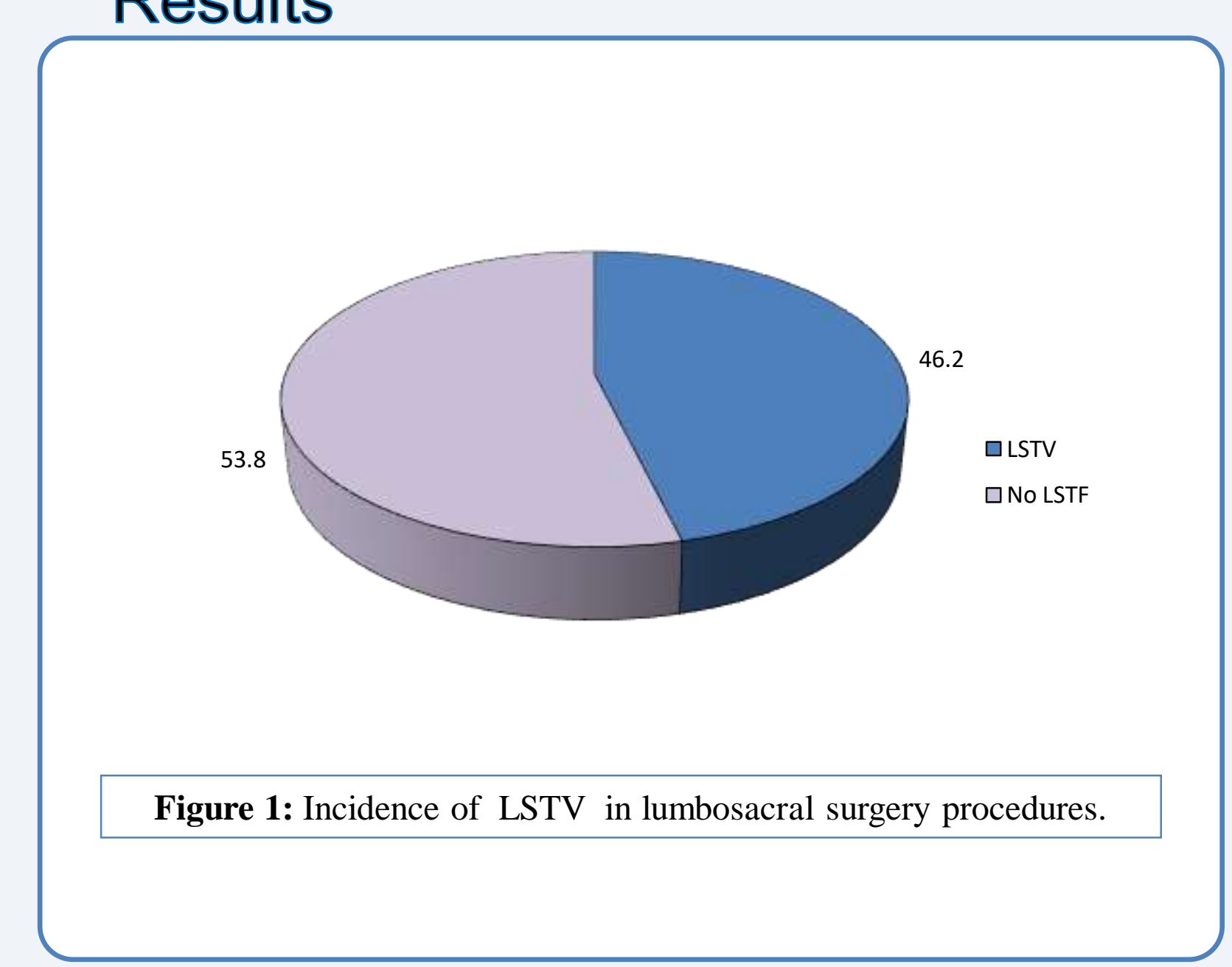

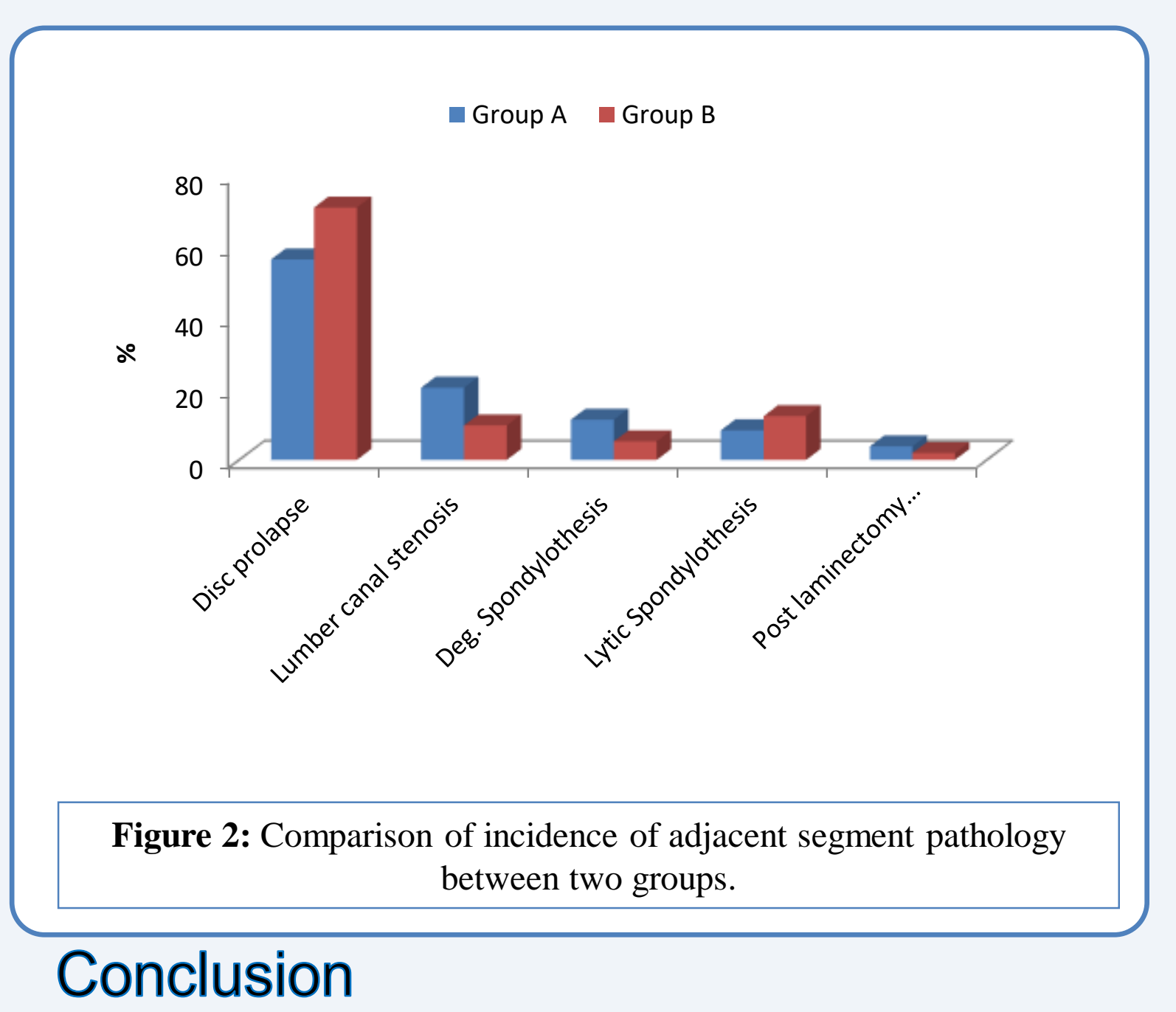

1. The overall incidence of LSTV among all cases that undergone lumbosacral surgical procedures at EL Hadra University Hospital is $46.2 \%$.

2. Incidence of lumbar canal stenosis, degenerative spondylolithesis is higher in LSTV group compared to non-LSTV group. However, incidence of disc prolapsed is lower in LSTV compared to non-LSTV.

3. LSTV is considered a risk factor for disc degenerative changes at the above level.

20210Alexandria Faculty of Medicine
CC-BY-NC

